\title{
Mammals of Gala Lake National Park
}

\author{
Beytullah ÖZKAN ${ }^{1} *$
}

ASTRACT: Gala Lake National Park (GLNP) consists of terrestrial and aquatic ecosystems covering 6.087 hectares of area in Enez and İpsala district boundaries of Edirne province. Extensive field studies were performed on mammals within the boundaries of GLNP for 26 days from 29 July to 07 November 2016. A total of 44 mammalian species from 16 families within 6 orders were determined in the studied area and an up-to-date inventory of mammals was prepared. Myocastor coypus (coypu) were detected as an invasive species in the region.

Keywords: Gala lake national park, mammalia, Enez, İpsala, Edirne, Thracia

\section{Gala Gölü Milli Parkı Memelileri}

ÖZET: Gala Gölü Milli Parkı Edirne iline bağlı Enez ve İpsala ilçe sınırları içerisinde yer alan, 6.087 ha alanı kaplayan karasal ve sulak ekosistemlerden oluşmaktadır. Gala Gölü Milli Parkı sınırları içerisinde memeli hayvanlar üzerine 29 Temmuz - 07 Kasım 2016 tarihleri arasında 26 günlük kapsamlı arazi çalışması gerçekleştirilmiştir. Bu çalışma sonunda memeli hayvanların güncel envanteri yapılarak Gala Gölü Milli Parkı sınırları içerisinde 6 takım, 16 familyaya ait toplam 44 memeli türünün bulunduğu tespit edilmiştir. Bölgede istilacı tür olarak Myocastor coypus (su maymunu) tespit edilmiştir.

Anahtar Kelimeler: Gala gölü milli parkı, memeli, Enez, İpsala, Edirne, Trakya 


\section{INTRODUCTION}

Gala Lake National Park (GLNP) is located in district borders of Enez and İpsala in Edirne and covers an area of 6.087ha including aquatic (stagnant water bodies and marshes) and terrestrial (forest and agricultural areas and meadows) ecosystems (Figure 1). The mammalian species living in and around GLNP have been determined with a numerous studies performed in the region (Spitzenberger, 1968; Kurtonur, 1972; Doğramac1, 1974; Ağşa, 1975; Kumerloeve, 1975; Kurtonur 1975; Kurtonur 1982; Turan, 1984; Özkan, 1987; Albayrak, 1988; Kıvanç, 1988; Kurtonur and Özkan, 1990; Kurtonur and Özkan, 1991; Kurtonur et al., 1994; Özkan and Kurtonur, 1994; Civitelli et al., 1995; Filippucci et al., 1995; Özkan, 1995; Kurtonur et al, 1996; Kryštufek et al., 1997; Benda and Horacek, 1998; Albayrak, 1999; Mitchell-Jones et al., 1999; Özkan, 1999; Özkan and Kryštufek, 1999; Çolak et al., 2000; Buruldağ and Kurtonur 2001; Kryštufek and Vohralik, 2001; Özkan et al., 2003; Yiğit et al., 2003; Çolak et al., 2005; Kryštufek and Vohralik, 2005; Özkan, 2006a; Özkan, 2006b; Yiğit et al., 2006; Paksuz et al., 2007; Paksuz and Özkan, 2008; İlker et al., 2009; Kryštufek and Vohralik, 2009; Kryštufek et al., 2009; Anonim, 2010a; Anonim, 2010b; Doğan, 2010; Paksuz and Özkan, 2011; Gruychev, 2012; Paksuz and Özkan, 2012; Anonim, 2014; Özkan and Paksuz, 2015; Anonim, 2016; Gruychev, 2017; Chloe and Legakis, 2016). However, a through investigation of some of these studies revealed that some mammals were lacking in the species lists reported and that some species which are hard to be present in the region were reported to occur here, showing the necessity of a detailed and updated inventory of mammals of the region.

The present study was performed in order to determine the current status of mammalian species present in aquatic and terrestrial ecosystems of GLNP. Field studies were performed in the region and the findings of these studies, in addition to the data obtained from previous literature, were used in preparation of an inventory to obtain an updated list of mammalian species living in the region.

\section{MATERIAL AND METHOD}

Different field study techniques depending on the type of the ecosystem monitored were used. Live traps were used for small sized mammals and photo traps (Bushnell Trophy Cam HD Aggressor Brown Model 119776) for used for medium and large sized mammals. Nets were used as the primary source of equipment for bats but a bioacoustics recording device (Wildlife Song Meter SM4BAT FS Bioaccustics Recorder) was also used for bats in the region for the first time. Species and their habitats were photographed and monitored using binoculars when possible. Individuals accidentally and/or naturally died along roads and/or in the field, nests, shelters, feces and any type of clues, e.g. hair, residues of eaten vegetation, left behind by travelling individuals were also considered. Previous studies and questionnaires with local people in the region were evaluated (see Figure $1)$.

The different ecosystem types in GLNP were visited during daytime and night to trap alive mammalian specimens with relevant techniques. The identifications of each individual were determined on site, their body size measurements were taken, biological and ecological notes were taken in detail and all were released in the field. The GPS data of sampling sites were also noted and determined species were evaluated considering their international and national conservation statuses.

\section{RESULTS AND DISCUSSION}

The field studies in GLNP and evaluation of available literature revealed presence of 44 mammalian species within 29 genera, 16 families in 6 orders (Tables 1 and 2). The distribution of the species with respect to families and orders were given in Tables 1 and 2 . 


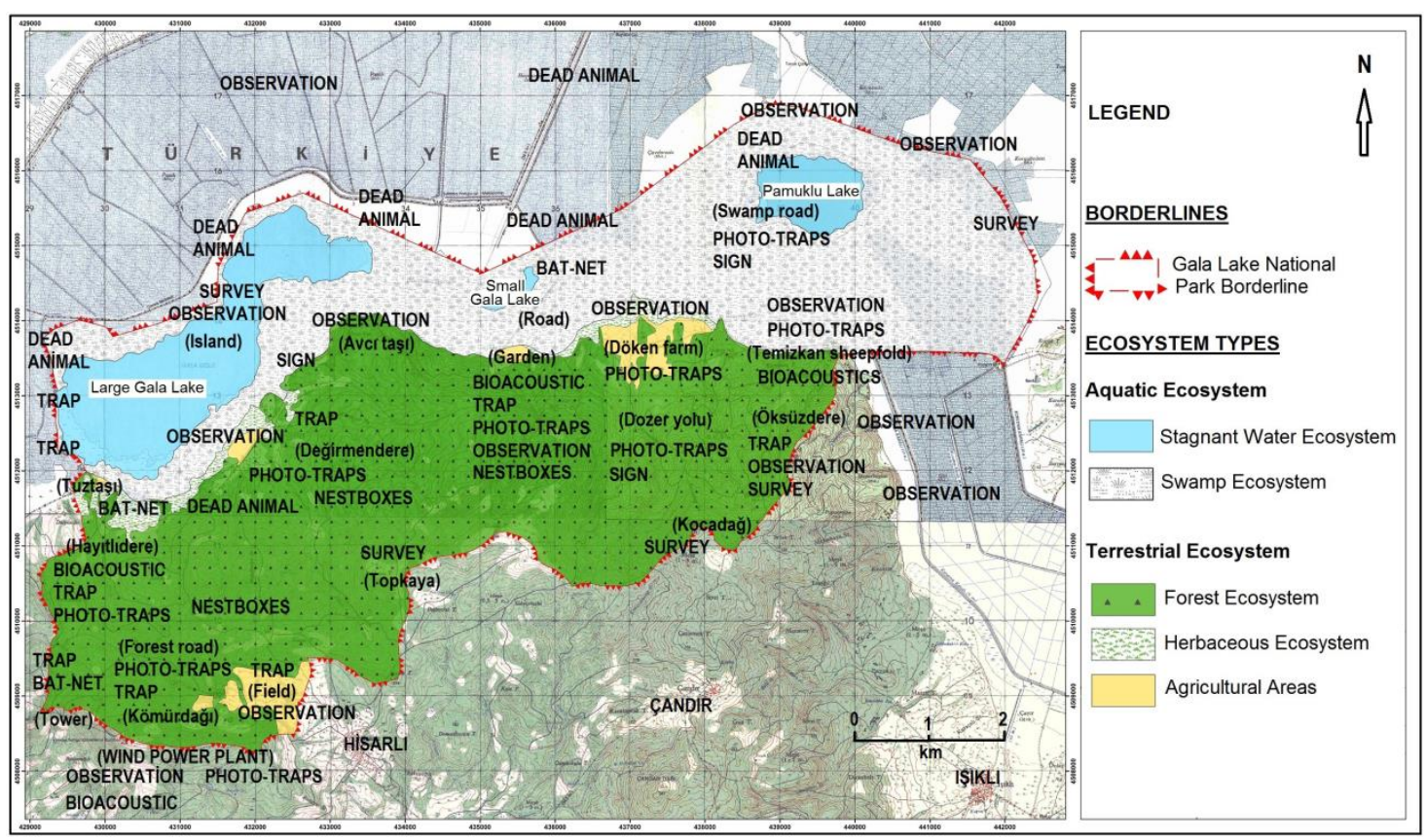

Figure 1.The localities visited in GLNP during the field studies. The techniques used to trap mammalian specimens were shown on the map (Modified from Anonymous (2016).

Table1. Mammals of GLNP and their international and national conservation statuses [MFWA= Ministry of Forestry and Water Affairs (App II = Specified Hunting Animals by the Ministry of Forestry and Water World, App III = Wild Animals Protected by the Ministry of Forestry and Water World)].

\begin{tabular}{|c|c|c|c|c|c|c|c|}
\hline Order & Family & Genus & Species & CITES & IUCN & BERN & MFWA \\
\hline $\begin{array}{ll}\text { 1- } & \text { EULIPOTYPHLA } \\
& \text { (Insectivorous) }\end{array}$ & 3 & 3 & 4 & - & $\mathrm{LC}=4$ & $\begin{array}{l}\text { APP-II }=1 \\
\text { APP-III }=1\end{array}$ & AppIII=4 \\
\hline 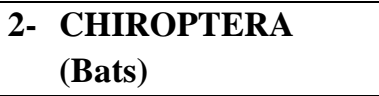 & 2 & 6 & 14 & - & $\begin{array}{l}\mathrm{LC}=11 \\
\mathrm{VU}=3\end{array}$ & $\begin{array}{l}\text { APP-II }=13 \\
\text { APP-III }=1\end{array}$ & AppIII=14 \\
\hline $\begin{array}{ll}\text { 3- } & \text { LAGOMORPHA } \\
& \text { (Rabbit and Hares) }\end{array}$ & 1 & 1 & 1 & - & $\mathrm{LC}=1$ & APP- III = 1 & - \\
\hline $\begin{array}{l}\text { 4- } \text { RODENTIA } \\
\text { (Rodents) }\end{array}$ & 6 & 10 & 14 & - & $\begin{array}{l}\mathrm{LC}=11 \\
\mathrm{VU}=1 \\
\mathrm{NT}=1 \\
\mathrm{DD}=1\end{array}$ & APP-III = 3 & AppIII $=5$ \\
\hline $\begin{array}{l}\text { 5- } \begin{array}{l}\text { CARNIVORA } \\
\text { (Carnivores) }\end{array} \\
\end{array}$ & 3 & 9 & 10 & $\begin{array}{l}\text { APP-1 }=1 \\
\text { APP-2 }=2\end{array}$ & $\begin{array}{l}\mathrm{LC}=8 \\
\mathrm{VU}=1 \\
\mathrm{NT}=1\end{array}$ & $\begin{array}{l}\text { APP-II }=4 \\
\text { APP-III }=4\end{array}$ & $\begin{array}{l}\text { App II }=4 \\
\text { AppIII }=6\end{array}$ \\
\hline $\begin{aligned} \text { 6- } & \text { ARTIODACTYLA } \\
& \text { (Even-toed ungulate) }\end{aligned}$ & 1 & 1 & 1 & - & $\mathrm{LC}=1$ & APP-III=1 & App II=1 \\
\hline Total & 16 & 30 & 44 & $\begin{array}{l}\text { APP-1=1 } \\
\text { APP-2 }=2\end{array}$ & $\begin{array}{l}\mathrm{LC}=\mathbf{3 6} \\
\mathrm{VU}=\mathbf{5} \\
\mathrm{NT}=\mathbf{2} \\
\mathrm{DD}=\mathbf{1}\end{array}$ & $\begin{array}{l}\text { APP-II }=18 \\
\text { APP-III }=11\end{array}$ & $\begin{array}{l}\text { App II=5 } \\
\text { AppIII=27 }\end{array}$ \\
\hline
\end{tabular}




\begin{tabular}{lc}
\hline Beytullah ÖZKAN & Journal of the Institute of Science and Technology, 9(2): 699-707, 2019 \\
\hline Mammals of Gala Lake National Park \\
\hline
\end{tabular}

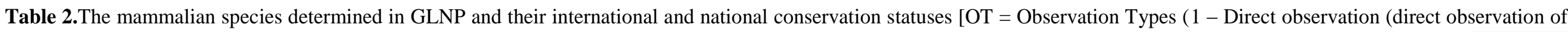

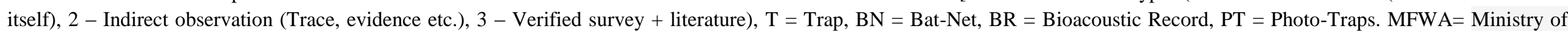

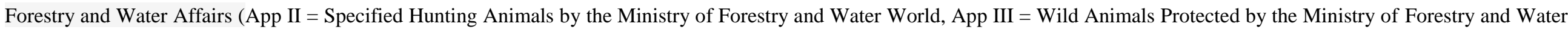
World)].

\begin{tabular}{|c|c|c|c|c|c|c|c|c|c|c|c|}
\hline Order & Family & $\begin{array}{l}\text { Common name in } \\
\text { Turkish }\end{array}$ & $\begin{array}{l}\text { Common name in } \\
\text { English }\end{array}$ & Scientific name & Density & Endemic & CITES & IUCN & BERN & MFWA & $* \mathbf{O T}$ \\
\hline \multirow{4}{*}{$\begin{array}{l}\text { EULIPOTYPHLA } \\
\text { (Insectivorous) }\end{array}$} & $\begin{array}{l}\text { Erinaceidae } \\
\text { (Hedgehogs) }\end{array}$ & Kirpi & $\begin{array}{l}\text { Northern white } \\
\text { breasted hedgehog }\end{array}$ & $\begin{array}{l}\text { Erinaceus roumanicus } \\
\text { Barret-Hamilton, } 1900\end{array}$ & Rare & - & - & $\mathrm{LC}$ & - & App III & 1 \\
\hline & $\begin{array}{r}\text { Talpidae } \\
\text { (Moles) }\end{array}$ & Köstebek & Common mole & $\begin{array}{l}\text { Talpa europaea } \\
\text { Linnaeus, } 1758\end{array}$ & Unknown & - & - & $\mathrm{LC}$ & - & App III & $1 ; 2$ \\
\hline & $\begin{array}{l}\text { Soricidae } \\
\text { (Shrews) }\end{array}$ & $\begin{array}{l}\text { Beyazdişli Küçük } \\
\text { Böçekcil }\end{array}$ & $\begin{array}{l}\text { Lesser white } \\
\text { toothed shrew }\end{array}$ & $\begin{array}{l}\text { Crocidura suaveolens } \\
\text { (Pallas, 1811) }\end{array}$ & Unknown & - & - & $\mathrm{LC}$ & APP II & App III & $\begin{array}{c}1 \\
(\mathrm{~T})\end{array}$ \\
\hline & & Beyazdişli Böçekcil & $\begin{array}{l}\text { Bi-coloured white- } \\
\text { toothed shrew }\end{array}$ & $\begin{array}{l}\text { Crocidura leucodon } \\
\text { (Hermann, 1780) }\end{array}$ & Unknown & - & - & $\mathrm{LC}$ & APP III & App III & $\begin{array}{c}1 \\
(\mathrm{~T})\end{array}$ \\
\hline \multirow{8}{*}{$\begin{array}{l}\text { CHIROPTERA } \\
\text { (Bats) }\end{array}$} & $\begin{array}{l}\text { Rhinolophidae } \\
\text { (Horseshoe } \\
\text { bats) }\end{array}$ & $\begin{array}{l}\text { Nalburunlu } \\
\text { Büyük yarasa }\end{array}$ & $\begin{array}{l}\text { Greater horseshoe } \\
\text { bat }\end{array}$ & $\begin{array}{l}\text { Rhinolophus } \\
\text { ferrumequinum } \\
\text { (Schreber, 1774) }\end{array}$ & Unknown & - & - & $\mathrm{LC}$ & APP III & App III & $\begin{array}{l}1,3 \\
(\mathrm{BN})\end{array}$ \\
\hline & & Mehely Yarasası & $\begin{array}{l}\text { Mehely'shorseshoe } \\
\text { bat }\end{array}$ & $\begin{array}{l}\text { Rhinolophus mehelyi } \\
\text { Matschie, } 1901\end{array}$ & Unknown & - & - & VU & APP II & App III & $\begin{array}{l}1 ; 3 \\
(\mathrm{BN})\end{array}$ \\
\hline & $\begin{array}{l}\text { Vespertilionidae } \\
\text { (Vesper bats) }\end{array}$ & Uzunayaklı Yarasa & Long-fingered bat & $\begin{array}{l}\text { Myotis capacciniii } \\
\text { (Bonaparte, 1837) }\end{array}$ & Unknown & - & - & VU & APP II & App III & $\begin{array}{l}1 ; 2 \\
(\mathrm{BN})\end{array}$ \\
\hline & & Su Yarasası & Dabenton's bat & $\begin{array}{l}\text { Myotis daubentoni } \\
\text { (Kuhl, 1817) }\end{array}$ & Unknown & - & - & $\mathrm{LC}$ & APP II & App III & $\begin{array}{l}1 ; 2 \\
(\mathrm{BR})\end{array}$ \\
\hline & & Beyazyakalı Yarasa & Kuhl's pipistrelle & $\begin{array}{l}\text { Pipistrellus kuhlii } \\
\text { (Kuhl, 1819) }\end{array}$ & Unknown & - & - & $\mathrm{LC}$ & APP II & App III & $\begin{array}{l}1 ; 2 \\
(\mathrm{BR})\end{array}$ \\
\hline & & $\begin{array}{l}\text { Pürtüklü Cüce } \\
\text { Yarasa }\end{array}$ & $\begin{array}{l}\text { Nathusius' } \\
\text { pipistrelle }\end{array}$ & $\begin{array}{l}\text { Pipistrellus nathusii } \\
\text { (Kayserling \& Blasius, } \\
\text { 1839) }\end{array}$ & Unknown & - & - & $\mathrm{LC}$ & APP II & App III & $\begin{array}{l}1 ; 2 \\
(\mathrm{BR})\end{array}$ \\
\hline & & $\begin{array}{l}\text { Bayağı Cüce } \\
\text { Yarasası }\end{array}$ & $\begin{array}{l}\text { Common } \\
\text { pipistrelle }\end{array}$ & $\begin{array}{l}\text { Pipistrellus pipistrellus } \\
\text { (Scheber, 1774) }\end{array}$ & Unknown & - & - & $\mathrm{LC}$ & APP II & App III & $\begin{array}{l}1 ; 2 \\
(\mathrm{BR})\end{array}$ \\
\hline & & $\begin{array}{l}\text { Akdeniz Cüce } \\
\text { Yarasa }\end{array}$ & Soprano pipistrelle & $\begin{array}{l}\text { Pipistrellus pygmaeus } \\
\text { (Leach, 1825) }\end{array}$ & Unknown & - & - & $\mathrm{LC}$ & APP II & App III & $\begin{array}{l}1 ; 2 \\
(\mathrm{BR})\end{array}$ \\
\hline
\end{tabular}




\begin{tabular}{|c|c|c|c|c|c|c|c|c|c|c|c|}
\hline Order & Family & $\begin{array}{l}\text { Common name in } \\
\text { Turkish }\end{array}$ & $\begin{array}{l}\text { Common name in } \\
\text { English }\end{array}$ & Scientific name & Density & Endemic & CITES & IUCN & BERN & MFWA & *OT \\
\hline & & $\begin{array}{l}\text { Büyük Akşamcı } \\
\text { Yarasa }\end{array}$ & Greater noctula & $\begin{array}{l}\text { Nyctalus lasiopterus } \\
\text { (Schreber, 1780) }\end{array}$ & Unknown & - & - & VU & APP II & App III & $\begin{array}{c}2 \\
(\mathrm{BR})\end{array}$ \\
\hline & & $\begin{array}{l}\text { Küçük Akşamc1 } \\
\text { Yarasa }\end{array}$ & Leisler's bat & $\begin{array}{l}\text { Nyctalus leisleri } \\
\text { (Kuhl, 1817) }\end{array}$ & Unknown & - & - & $\mathrm{LC}$ & APP II & App III & $\begin{array}{c}2 \\
(\mathrm{BR})\end{array}$ \\
\hline & & Akşamcı Yarasa & Noctula & $\begin{array}{l}\text { Nyctalus noctula } \\
\text { (Schreberi 1774) }\end{array}$ & Unknown & - & - & $\mathrm{LC}$ & APP II & App III & $\begin{array}{c}2 \\
(\mathrm{BR})\end{array}$ \\
\hline & & Genişkanatlı Yarasa & Serotina & $\begin{array}{l}\text { Eptecicus serotinus } \\
\text { Schreber, } 1774\end{array}$ & Unknown & - & - & $\mathrm{LC}$ & APP II & App III & $\begin{array}{c}2 \\
(\mathrm{BR})\end{array}$ \\
\hline & & $\begin{array}{l}\text { Kahverengi } \\
\text { Uzunkulakl1 Yarasa }\end{array}$ & $\begin{array}{l}\text { Brown long-eared } \\
\text { bat }\end{array}$ & $\begin{array}{l}\text { Plecotus auritus } \\
\text { (Linnaeus, 1758) }\end{array}$ & Unknown & - & - & $\mathrm{LC}$ & APP II & App III & $\begin{array}{c}2 \\
(\mathrm{BR})\end{array}$ \\
\hline & & $\begin{array}{l}\text { Kahverengi } \\
\text { Uzunkulaklı Yarasa }\end{array}$ & $\begin{array}{l}\text { Grey long-eared } \\
\text { bat }\end{array}$ & $\begin{array}{l}\text { Plecotusa austriacus } \\
\text { (Linnaeus, 1758) }\end{array}$ & Unknown & - & - & $\mathrm{LC}$ & APP II & App III & 3 \\
\hline $\begin{array}{l}\text { LAGOMORPHA } \\
\text { (Rabbits and Hares) }\end{array}$ & $\begin{array}{l}\text { Leporidae } \\
\text { (Hares) }\end{array}$ & $\begin{array}{l}\text { Kur Tavşanı, } \\
\text { Yabani Tavşan } \\
\end{array}$ & Brown hare & $\begin{array}{l}\text { Lepus europaeus } \\
\text { (Pallas, 1778) }\end{array}$ & Middle & - & - & $\mathrm{LC}$ & APP III & App II & $\begin{array}{l}1 ; 2 \\
(\mathrm{PT}) \\
\end{array}$ \\
\hline \multirow{10}{*}{$\begin{array}{l}\text { RODENTIA } \\
\text { (Rodents) }\end{array}$} & $\begin{array}{l}\text { Sciuridae } \\
\text { (Squirrels) }\end{array}$ & Avrupa Sincab1 & Red squirrel & $\begin{array}{l}\text { Sciurus vulgaris } \\
\text { Linnaeus, } 1758\end{array}$ & Rare & -- & - & $\mathrm{LC}$ & APP III & App III & $1 ; 3$ \\
\hline & & $\begin{array}{l}\text { Gelengi, } \\
\text { Kazıksıçanı }\end{array}$ & European souslik & $\begin{array}{l}\text { Spermophilus citellus } \\
\text { (Linnaeus, 1766) }\end{array}$ & Middle & -- & - & VU & APP III & App III & 1 \\
\hline & $\begin{array}{l}\text { Cricetidae } \\
\text { (Voles) }\end{array}$ & $\begin{array}{l}\text { Uzunkuyruklu } \\
\text { Çayır Faresi }\end{array}$ & Sibling vole & $\begin{array}{l}\text { Microtus levis } \\
\text { Ognev, } 1924\end{array}$ & Unknown & - & - & $\mathrm{LC}$ & & - & 2 \\
\hline & & Tarla Faresi & Guenther's vole & $\begin{array}{l}\text { Microtus guentheri } \\
\text { (Danford and Alston, } \\
1880 \text { ) }\end{array}$ & Unknown & - & - & $\mathrm{LC}$ & & - & 2 \\
\hline & $\begin{array}{l}\text { Spalacidae } \\
\text { (Mole rat) }\end{array}$ & Körfare & Lesser mole rat & $\begin{array}{l}\text { Nannospalax leucodon } \\
\text { (Nordmann, 1840) }\end{array}$ & Common & - & - & DD & & - & $1 ; 3$ \\
\hline & $\begin{array}{l}\text { Muridae } \\
\text { (Rats and mice) }\end{array}$ & Hasat Faresi & Harvest mouse & $\begin{array}{l}\text { Micromys minutus } \\
\text { (Pallas, 1771) }\end{array}$ & Unknown & - & - & NT & & App III & $1 ; 3$ \\
\hline & & Orman Faresi & $\begin{array}{l}\text { Yellow-necked } \\
\text { mouse }\end{array}$ & $\begin{array}{l}\text { Apodemus flavicollis } \\
\text { (Melchior, 1834) }\end{array}$ & Common & - & - & $\mathrm{LC}$ & & - & $\begin{array}{l}1 ; 2 \\
(\mathrm{~T})\end{array}$ \\
\hline & & Tarla faresi & Wood mouse & $\begin{array}{l}\text { Apodemus sylvaticus } \\
\text { (Linnaeus, 1758) }\end{array}$ & Common & - & - & $\mathrm{LC}$ & & - & $\begin{array}{c}1 \\
(\mathrm{~T})\end{array}$ \\
\hline & & Ev Siçanı & Black rat & $\begin{array}{l}\text { Rattus rattus } \\
\text { (Linnaeus, 1758) }\end{array}$ & Rare & - & - & $\mathrm{LC}$ & - & - & $1 ; 3$ \\
\hline & & Göçmen Siçan & Brown rat & $\begin{array}{l}\text { Rattus norvegicus } \\
\text { (Berkenhout, 1769) }\end{array}$ & Rare & -- & - & $\mathrm{LC}$ & - & -- & $\begin{array}{l}1 ; 3 \\
(\mathrm{~T})\end{array}$ \\
\hline
\end{tabular}




\begin{tabular}{|c|c|c|c|c|c|c|c|c|c|c|c|}
\hline Order & Family & $\begin{array}{l}\text { Common name in } \\
\text { Turkish }\end{array}$ & $\begin{array}{l}\text { Common name in } \\
\text { English }\end{array}$ & Scientific name & Density & Endemic & CITES & IUCN & BERN & MFWA & $* \mathbf{O T}$ \\
\hline & & $\begin{array}{l}\text { Makedonya } \\
\text { Ev Faresi }\end{array}$ & $\begin{array}{l}\text { Balkan short-tailed } \\
\text { mouse }\end{array}$ & $\begin{array}{l}\text { Mus macedonicus } \\
\text { Petrov \& Ruzic, 1983) }\end{array}$ & Middle & - & - & $\mathrm{LC}$ & - & - & $\begin{array}{c}1 \\
(\mathrm{~T}) \\
\end{array}$ \\
\hline & & Ev faresi & $\begin{array}{l}\text { Western house } \\
\text { mouse }\end{array}$ & $\begin{array}{l}\text { Mus domesticus } \\
\text { Rutty, } 1772 \\
\end{array}$ & Rare & - & - & $\mathrm{LC}$ & - & - & $\begin{array}{c}1 \\
(\mathrm{~T})\end{array}$ \\
\hline & $\begin{array}{l}\text { Gliridae } \\
\text { (Dormouses) }\end{array}$ & $\begin{array}{l}\text { Ağaç Faresi, } \\
\text { Cevizkıran }\end{array}$ & Forest mouse & $\begin{array}{l}\text { Dryomys nitedula } \\
\text { (Pallas, 1779) }\end{array}$ & Middle & - & - & $\mathrm{LC}$ & APP III & App III & $\begin{array}{c}1 \\
(\mathrm{~T})\end{array}$ \\
\hline & $\begin{array}{l}\text { Myocastoridae } \\
\text { (Coypus) }\end{array}$ & Su Maymunu & Coypu & $\begin{array}{l}\text { Myocastor coypus } \\
\text { (Molina, 1758) }\end{array}$ & Common & - & - & $\mathrm{LC}$ & - & App III & $\begin{array}{c}1 \\
(\mathrm{PT})\end{array}$ \\
\hline \multirow{10}{*}{$\begin{array}{l}\text { CARNIVORA } \\
\text { (Carnivores) }\end{array}$} & Canidae (Canids) & Kurt & Wolf & $\begin{array}{l}\text { Canis lupus } \\
\text { Linnaeus, } 1758\end{array}$ & Unknown & - & APP 2 & $\mathrm{LC}$ & APP II & App III & 3 \\
\hline & & Çakal & Golden jackal & $\begin{array}{l}\text { Canis aureus } \\
\text { Linnaeus, } 1758\end{array}$ & Middle & - & - & $\mathrm{LC}$ & - & App II & $\begin{array}{l}2 ; 3 \\
(\mathrm{PT})\end{array}$ \\
\hline & & Tilki & Vulpes & $\begin{array}{l}\text { Vulpes vulpes } \\
\text { (Linnaeus, 1758) }\end{array}$ & Common & - & - & $\mathrm{LC}$ & - & App II & $\begin{array}{c}1 ; 2 ; 3 \\
(\mathrm{PT})\end{array}$ \\
\hline & $\begin{array}{r}\text { Mustelidae } \\
\text { (Martens) }\end{array}$ & Kaya Sansarı & $\begin{array}{l}\text { Beech marten, Stone } \\
\text { marten }\end{array}$ & $\begin{array}{l}\text { Martes foina } \\
\text { (Erxleben, 1777) }\end{array}$ & Middle & - & - & $\mathrm{LC}$ & APP III & App II & $\begin{array}{c}2 \\
(\mathrm{PT})\end{array}$ \\
\hline & & Porsuk & Badger & $\begin{array}{l}\text { Meles meles } \\
\text { (Linnaeus, 1758) }\end{array}$ & Middle & - & - & $\mathrm{LC}$ & APP III & App II & $\begin{array}{c}2 \\
(\mathrm{PT})\end{array}$ \\
\hline & & Su Samuru & Otter & $\begin{array}{l}\text { Lutra lutra } \\
\text { (Linnaeus, 1758) }\end{array}$ & Rare & - & APP 1 & NT & APPII & App III & $\begin{array}{c}2 \\
(\mathrm{PT})\end{array}$ \\
\hline & & Gelincik & Weasel & $\begin{array}{l}\text { Mustela nivalis } \\
\text { Linnaeus, } 1766 \\
\end{array}$ & Rare & - & - & $\mathrm{LC}$ & APP III & App II & 3 \\
\hline & & Kokarca & Western polecat & $\begin{array}{l}\text { Mustela putorius } \\
\text { Linnaeus, } 1758 \\
\end{array}$ & Rare & - & - & $\mathrm{LC}$ & APP III & App II & 3 \\
\hline & & Alaca Kokarca & Marbled polecat & $\begin{array}{l}\text { Vormela peregusna } \\
\text { (Guldenstaedt, 1770) }\end{array}$ & Unknown & - & - & VU & APP II & App III & 3 \\
\hline & Felidae (Cats) & Yaban Kedisi & Wildcat & $\begin{array}{l}\text { Felis silvestris } \\
\text { Schreber, } 1777\end{array}$ & Rare & - & APP 2 & $\mathrm{LC}$ & APP II & App III & $\begin{array}{l}1 ; 2 \\
(\mathrm{PT})\end{array}$ \\
\hline $\begin{array}{l}\text { ARTIODACTYLA } \\
\text { (Even-toed ungulate) }\end{array}$ & Suidae (Pigs) & Yaban Domuzu & Wildboar & $\begin{array}{l}\text { Sus scrofa } \\
\text { Linnaeus, } 1758\end{array}$ & Common & - & - & $\mathrm{LC}$ & - & App II & $\begin{array}{l}1 ; 2 \\
(\mathrm{PT})\end{array}$ \\
\hline 6 & 16 & & & 30 genera and 44 species & $\begin{array}{c}\text { Rare }=9 \\
\text { Middle }=7 \\
\text { Common }=6 \\
\text { Unknown }=22\end{array}$ & - & $\begin{array}{l}\text { APP } 1=1 \\
\text { APP } 2=2\end{array}$ & $\begin{array}{l}\mathrm{LC}=36 \\
\mathrm{VU}=4 \\
\mathrm{NT}=3 \\
\mathrm{DD}=1\end{array}$ & $\begin{array}{l}\text { APP II }=18 \\
\text { APP III }=11\end{array}$ & $\begin{array}{c}\text { App II }=5 \\
\text { App III }=29\end{array}$ & \\
\hline
\end{tabular}




\section{CONCLUSION}

The mammalian fauna of Turkey is represented with 172 species and 70 of these species $(40.1 \%)$ lives in Thrace region, 52 $(30.2 \%)$ in provincial borders of Edirne and 44 (25.5\%) in GLNP (Kumerloeve, 1975; Özkan, 1987; Kurtonur et al., 1996; Kryštufek et al., 1997; Mitchell-Jones et al., 1999; Kryštufek and Vohralik, 2001; Kryštufek and Vohralik, 2005; Çolak et al., 2005; Yiğit et al., 2006; Kryštufek and Vohralik, 2009; Kryštufek et al., 2009; Anonim, 2010a; Anonim, 2010b; Anonim, 2014; Anonim, 2016).

The international and national conservation statuses of these 44 species were given in Table 2.

Apodemus flaviollis (yellow-necked mouse) is one of the most common small mammal seen during the field studies in the terrestrial ecosystems (Doğramac1, 1974; Çolaket al., 2005; Anonim, 2014; Anonim, 2016).

Myocastor coypus (coypu) was determined to be an invasive species in GLNP (Özkan, 1999; Anonim, 2016). The population size of this species in Maritza River basin, GLNP and related wetlands such as lakes, ponds, streams, channels etc. was determined to increase leading to economic and ecological losses in agricultural rice fields and wetlands by consuming rice seedlings, reeds and aquatic plants in huge amounts. The harm given to wetland vegetation indirectly and irreversibly affected bird nesting and breeding sites and breeding areas of fish populations in lakes.

A detailed study on impacts of coypu in GLNP is planned to be implemented in near future.

The questionnaires showed that the roe deer Capreolus capreolus was completely removed from the region by 1984 as a result of hunting activities and habitat destructions.

Personal observations in the region during the study showed that a huge amount of grazing due to stock farming and sheep and goat farming is taking place.

The region located underside parts of wind power plants established in Hisar Mountain in the southern border of GLNP were monitored for the presence of dead bats but no death individual was encountered during the monitoring leading us to conclude that the wind turbines used had no negative effect on bats of GLNP.

\section{REFERENCES}

Ağşa L, 1975. Su Maymunu. Av Dergisi 8: 3-4.

Albayrak İ, 1988. Batı Türkiye Yarasaları ve Yarasa Pireleri. Türkiye Bilimsel ve Teknik Araştırma Kurumu. TBAG-663. 80 sayfa.

Albayrak İ, 1999. Fauna: Su samuru, ATLASYeşil Atlas, 2: 82-83.

Anonim 2010a. Gala Gölü Milli Parkı Uzun Devreli Gelişme Planı Analitik Etüt Raporu. T.C. Orman ve Su İşleri Bakanlığı, Doğa Koruma ve Milli Parklar Genel Müdürlüğü. Belde Proje ve Danışmanlık Ticaret Limited Şirketi - Ankara. 581 sayfa.

Anonim 2010b. Gala Gölü Milli Parkı Uzun Devreli Gelişme Planı Sentez Raporu. T.C. Orman ve Su İşleri Bakanlığı, DoğaKoruma ve Milli Parklar Genel Müdürlüğü. Belde Proje ve Danışmanlık Ticaret Limited Şirketi - Ankara. 70 sayfa.

Anonim 2014. Edirne İlinin Karasal ve İç Su Ekosistemleri Biyolojik Çeşitlilik Envanter ve İzleme Projesi. Orman ve Su İşleri III. Bölge Müdürlüğü / Edirne Şube Müdürlüğü, Edirne. 783 sayfa.

Anonim 2016. Gala Gölü Milli Parkı'nın Flora, Fauna ve Vejetasyon Tespiti. T.C. Orman ve $\mathrm{Su}$ İşleri Bakanlığı Doğa Koruma ve Milli Parklar Müdürlüğü I. Bölge Müdürlüğü, Edirne Şube Müdürlüğü. 265 sayfa.

Benda P, Horacek I, 1998. Bats (Mammalia: Chiroptera) of the Eastern Mediterranean. Part 1. Review of distribution and taxonomy of bats in Turkey. Acta Societatis Zoologicae Bohemicae 62: 255-313.

Buruldağ E, Kurtonur C, 2001. Hibernation and Postnatal Development of the Mouse-Tailed Dormouse, Myomimus roachi Reared Outdoor's in a Cage. Trakya University Journal of Scientific Research Series B, 2 (2): 179-186. 
Chloe A, Legakis A, 2016. First account on the occurrence of selected invasive alien vertebrates in Greece. Bio Invasions Records, 5 (4): 189-196.

Civitelli MV, Filuppucci MG, Kurtonur C, Özkan B,Capanna E, 1995. Chromosome analysis of three species of Myoxidae. In: Flipppucci M. G. (ed). Proc. II. Conf. On Dormice. Hystrix (n.s.) 6 (1-2) : 117 - 126.

Çolak E, Yiğit N, Sözen M, Verimli R, Özkurt ÖŞ, 2000. On coloration and karyology of Vormela peregusna, in Turkey, Zoology in the Middle East, 21: 13-18.

Çolak R, Çolak E, Yiğit N, 2005. Morphometric, Karyotypic and Electrophoretic Analysis of the Genus Apodemus Kaup, 1826 (Mammalia: Rodentia) in Thrace. Turkish Journal of Zoology, 29 (2): 147-153.

Doğan M, 2010. Trakya'da Yayılış Gösteren Microtus (Mammalia; Rodentia) Türlerinin Morfolojik Özellikleri. Ankara Üniversitesi, Fen Bilimleri Enstitüsü 1-57. Ankara (Yüksek Lisans Tezi).

Doğramac1 S, 1974. Türkiye Apodemus (Mammalia; Rodentia)'larının Taksonomik Durumları. Tarım Hayvancılık Bakanlığ Zirai Mücadele Müdürlügü Araştırma, 1-56.

Filippucci MG, Krystufek B, Simson S, Kurtonur C,Özkan B, 1995. Allozymic and Biometric Variation in Dryomys nitedula (Pallas, 1778). In: Filippuci M.G. (ed.). Proc. II Conf. on Dormice. Hystrix. (n.s.) 6 (1-2): $127-140$.

Gruychev G, 2012. New Record of Nutria (Myocastor coypus (Molina, 1782)) Downstream of The Maritsa River in Bulgaria. Forestry Ideas vol. 18 (1): 110112.

Gruychev G, 2017. Distribution And Density Of Coypu (Myocastor Coypus (Molina, 1782)) In Downstream Of Maritsa River Southeast Bulgaria. Forestry Ideas, 23 (1): 77-81.

İlker A, Arslan A, Pamukoğlu N, Albayrak İ, 2009. C-Banded karyotype of Myocastor coypus (Molina, 1782) from Turkey (Mammali: Rodentia). Folia Biologica, 57: 33-36.

Kıvanç E, 1988. Türkiye Spalax'larının Coğrafik Varyasyonları (Mammalia; Rodentia). Ankara Üniversitesi, Fen Fakültesi, Biyoloji Bölümü, 88 pp. (Doktora tezi).
Kryštufek B, Vohralik V, 2001. Mammals of Turkey and Cyprus. Introduction, Checlist, Insectivora. Knjiznica Anneles Majora. 140 pp. Koper, Slovenia.

Kryštufek B, Vohralik V, 2005. Mammals of Turkey and Cyprus. Rodentia I: Sciuridae, Dipodidae, Gliridae, Arvicolinae. Knjiznica Anneles Majora. 292 page, Koper, Slovenia.

Kryštufek B, Vohralik V, 2009. Mammals of Turkey and Cyprus. Rodentia II: Cricetinae, Muridae, Spalacidae, Calomyscidae, Capromyidae, Hysticidae, Castoridae. Knjiznica Anneles Majora, 372 pp. Koper, Slovenia.

Kryštufek B, Murariu D, Kurtonur C, 1997. Present distribution of the Golden Jackal Canis aureus in the Balkans and adjacent regions. Mammal Review, 27(2): 109-114.

Kryštufek B, Tvrtkovic N, Paunovic M, Özkan B, 2009. Size variation in the Northern whitebreasted hedgehog Erinaceus roumanicus: latitudinal cline and the island rule. Mammalia, 73: 299-306.

Kumerloeve H, 1975. Die Saugetierte (Mammalia) der Turkei. Veröffentlichungen der Zoologischen Staatssamlung München, 18: 69-158.

Kurtonur C, 1972. Trakya Rodentia'ları Üzerinde Taksonomik Bir Araştırma. İstanbul Üniversitesi. 69 sayfa. (Doktora Tezi).

Kurtonur C, 1975. New Records of Thracian Mammals. Säugetierkundlische Mitteilungen, 23 (1): 14 - 16.

Kurtonur C, 1982. Trakya Glirid Türleri (Rodentia; Gliridae). -Dağılımı, Habitat, Taksonomik Karekterler- İstanbul Üniversitesi. 70 Sayfa (Doçentlik Tezi).

Kurtonur C, Kryštufek B, Özkan B, 1994. The European polecat (Mustela putorius) In Turkish Thrace. Small Carnivore Conservation. (IUCN), 11: $8-10$.

Kurtonur C, Özkan B, 1990. Orman Ağaç Faresi Dryomys nitedula (Rodentia: Gliridae)'nın Trakya'daki Dağılımı ve Üreme Mevsimi. X. Ulusal Biyoloji Kongresi, 18 - 20 Temmuz 1990, Erzurum.

Kurtonur C, Özkan B, Albayrak İ, Kıvanç E, Kefelioğlu H, 1996. Memeliler (Mammalia); Türkiye Omurgalılar Tür Listesi. Editör: Kence, A., Bilgin, C. C. : DPT/TBAG Çevre Seksiyonu 3. 1-23. Ankara. 
Kurtonur C, Özkan B, 1991. New Records of Myomimus roachi (Bate, 1937) From Turkish Thrace (Mammalia: Rodentia: Gliridae). Senckenbergiana Biologica, 71: 239 - 244.

Mitchell-Jones AJ, Amori G, Bogdanowicz W, Kryštufek B, Reijnders PJH, Spitzenberger F, Stubbe M, Thissen JBM, Vohralik V, Zima J, 1999. The Atlas of European Mammals. The Academic Press, London. $496 \mathrm{pp}$.

Özkan B, Kryštufek B, 1999. Wood Mice, Apodemus of Two Turkish Island: Gökçeada and Bozcaada, Folia Zoologica, 48 (1): 1724.

Özkan B, Kurtonur C, 1994. Trakya Bölgesi'nde Myocastor coypus (Molina, 1782) (Su maymunu) (Rodentia: Mammalia)'a Ait İlk Kayıt. XII. Ulusal Biyoloji Kongresi, 6 - 8 Temmuz 1994, Edirne.

Özkan B, Paksuz S, 2015. Yeryediuyuru (Myomimus roachi) Tür Koruma Eylem Planı. Orman ve Su İşleri Bölge Müdürlügü Edirne Şube Müdürlüğü. 110 sayfa.

Özkan B, 1987. Edirne Kemiricileri. Trakya Üniversitesi, Fen Bilimleri Enstitüsü, Biyoloji Anabilim Dalı. 39 sayfa (Yüksek Lisans Tezi).

Özkan B, 1995. Gökçeada ve Bozcaada Adalarının Kemiricileri. Trakya Üniversitesi, Fen Bilimleri Enstitüsü, Biyoloji Anabilim Dalı. 156 sayfa (Doktora Tezi).

Özkan B, 1999. Invasive coypus, Myocastor coypus (Molina; 1782) in European part of Turkey. Israel Journal of Zoology, 45: 289291.

Özkan B, 2006a. An Observation on the Reproductive Biology of Glis glis (Linnaeus, 1766) (Rodentia; Gliridae) and Body Weight Gaining of Pups in the Istranca Mountains of Turkish Thrace. International Journal of Zoological Research, 2 (2): 129-135.

Özkan B, 2006b. Trakya Bölgesinde Yaban Kedisi (Felis silvestris Schreber, 1775)' in Dağılımı. 18. Ulusal Biyoloji Kongresi, 26-30 Haziran 2006, AYDIN.
Özkan B, Yiğit, N., Çolak, E. 2003. A study on Micromys minutus Pallas, 1771 (Mammalia: Rodentia) in Turkish Thrace. Turkish Journal of Zoology. 27: 55-60.

Paksuz S. Özkan B, 2008. Istranca OrmanlarınınYarasa Faunasi. XIX.Ulusal Biyoloji Kongresi, 23-27 Haziran 2008, Trabzon.

Paksuz S, Özkan B, 2011. New Distribution Records and Some Notes for Greater Noctule, Nyctalus lasiopterus (Mammalia: Chiroptera) from Turkey. Acta Zoologica Bulgarica, 63 (2): 217-220.

Paksuz S, Özkan B, 2012. The protection of the bat community in the Dupnisa Cave System, Turkey, following opening for tourism. Fauna \& Flora International, Oryx, 46 (1): 130-136.

Paksuz S, Özkan B, Postawa T, 2007. Seasonal Changes of Cave-dwelling Bat Fauna and Their Relationship with Microclimate in Dupnisa Cave System (Turkish Thrace). Acta Zoologica Cracoviensia, Serias A: Vertebrata, 50 (1-2): 57-66.

Spitzenberger F, 1968. Zur Verbreitung und Systematik Türkischer Soricinae (Insectivora, Mamm.). Annalen des Naturhistorischen Museums in Wien, 72: 273-289.

Turan N, 1984. Türkiye'nin Av ve Yaban Hayvanları - Memeliler. Ongun Kardeşler Matbaacılık Sanayii, Ankara, 178 sayfa.

Yiğit N, Çolak E, Çolak R, Özkan B, Özkurt ÖŞ, 2003. On the Turkish Populations of Dryomys nitedula (Pallas, 1779) and Dryomys laniger Felten and Storch, 1968 (Mammalia: Rodentia). Acta Zoologica Academiae Scientiarum Hungaricae 49 (Supl. 1), pp. 147-158.

Yiğit N, Çolak E, Sözen M, Karataş A, 2006. Rodents of Türkiye (Türkiye Kemiricileri). (Ed. Ali Demirsoy). Meteksan, Ankara. 1-15 